\title{
SISTEMA DE EVALUACIÓN REDER Y EL PLAN ESTRATÉGICO DE UNA INSTITUCIÓN PRIVADA CONFESIONAL
}

\author{
Miluska Villar Guevara ${ }^{1 \mathrm{a}}$ y Gladys Toyohama Pocco $^{2}$ \\ Universidad Peruana Unión, Lima, Perú ${ }^{12}$ \\ Universidad ESAN, Lima, Perú ${ }^{2}$ \\ Orcid ID: https://orcid.org/0000-0002-9632-5293 ${ }^{1}$ \\ Orcid ID: https://orcid.org/0000-0003-0118-3470²
}

\begin{abstract}
Resumen
Este artículo tiene el objetivo de describir el nivel de cumplimiento del plan estratégico a través del sistema de evaluación REDER de una institución privada confesional, 2017-2019. La metodología utilizada es descriptiva, de diseño no experimental, de corte trasversal, elaborada bajo un enfoque cuantitativo. Al interpretar estadísticamente los resultados se empleó el Software SPSS 24 para análisis descriptivo de frecuencia y de tablas cruzadas. Se aplicó un instrumento de 26 reactivos, dividido en 5 dimensiones, el cual fue administrado a una población de 45 personas de la administración. Se pudo comprobar que el $40 \%$ del total de la población de estudio, percibe niveles muy altos y notables de cumplimiento; sin embargo, el $35.6 \%$ consideraba el no cumplimiento de estos parámetros. Se concluye que el nivel de cumplimiento del plan estratégico a través del sistema de evaluación REDER, se ubica por debajo de los estándares medios; lo cual se traduce como un punto de mejora en lo que refiere a los patrones de un plan estratégico.
\end{abstract}

Palabras clave: Plan estratégico, sistema de evaluación REDER, modelo EFQM de excelencia, planificación estratégica.

\section{REDER EVALUATION SYSTEM AND THE STRATEGIC PLAN OF A CONFESSIONAL PRIVATE INSTITUTION}

\begin{abstract}
This article aims to describe the level of compliance of the strategic plan through the REDER evaluation system of a private confessional institution, from 2017-2019. The methodology used is descriptive, non-experimental, cross-sectional, and elaborated under a quantitative approach. When interpreting the results statistically, the SPSS 24 Software was used for descriptive analysis of frequency and cross-tabulations. An instrument of 26 items was applied, divided into 5 dimensions, which was administered to a population of 45 people in the administration. It was found that $40 \%$ of the total study population perceives very high and notable levels of compliance; however, $35.6 \%$ registered non-compliance with these parameters. It is concluded that the level of compliance with the strategic plan through the REDER evaluation system is
\end{abstract}

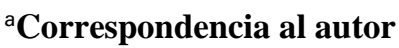

E-mail: miluskavillar@gmail.com 
below the average standards; which translates to a point of improvement in regards to the patterns of a strategic plan.

Keywords: Strategic plan, REDER evaluation system, EFQM model of excellence, strategic planning.

\section{Introducción}

El plan estratégico se ha convertido en una de las herramientas más importantes de las organizaciones que aprenden; y para su aplicación y desarrollo se requiere de un sistema de evaluación a fin de verificar sus estándares. Éste, también se considera como un instrumento que logra el perfeccionamiento de las capacidades dentro de una empresa (Araya, 2017). Por consiguiente, el presente estudio tuvo la finalidad de describir el nivel de cumplimiento del plan estratégico a través del sistema de evaluación REDER de una institución privada confesional, 2017-2019. La planificación estratégica es considerada un factor clave en una organización; y responde a las necesidades de un mundo cambiante y lo eleva a un nivel de competitividad mayor (Mendoza, López, \& Salas, 2016). Su desarrollo ha ido en continuo crecimiento a tal punto que se han destacado estudios empíricos integrando factores tecnológicos con el fin de hacerlo aún más efectivo (Martelo, Ponce, \& Acuña, 2016).

Diversos estudios describen a la planeación estratégica como un elemento fundamental en el crecimiento y el sostén futuro de las organizaciones. Existen empresas que según su composición se consolidan como una valiosa posibilidad de mejoramiento en su competitividad, es por ello que se deben usar todas las herramientas efectivas con el fin de superar los problemas que la puedan estar afectando (Mora-Riapira, Vera-Colina, \& MelgarejoMolina, 2015). Por otro lado, la crisis que enfrenta el mundo social, económico y empresarial nos empuja a pensar en modos estratégicos para luego ser llevados a la práctica y estos nos encaminen hacia coordenadas de conversión y reforma, el cual es impulsado por una planificación estratégica efectiva (Zavala-Mendoza \& Varela, 2017).

La variada información que existe sobre planes estratégicos debe ser capitalizada por las empresas y así evitar que sus administradores se resistan a ser evaluados, y, por supuesto, saber si el plan estratégico que se sigue está dando los resultados esperados por los socios, accionistas y la alta dirección. Una planificación estratégica bien ejecutada requiere el compromiso desde los altos mandos hasta el nivel más básico (Gonzales \& Gonzales, 2017). En muchos casos, la intransigencia de los administradores al evitar la evaluación conlleva a que la organización no perciba si el sistema de planificación estratégica está produciendo los resultados que se espera (Gutiérrez, Alizo, Morales, \& Romero, 2016; Pérez-Vallejo, Vilariño-Corella, \& Ronda-Pupo, 
2016; Sánchez, 2017). Un instrumento de evaluación como herramienta destinada a registrar información y datos básicos de una compañía, documentando el desempeño de las personas, permite verificar los resultados de trabajos obtenidos de acuerdo con normas y parámetros previamente definidos por la organización (Jaimes, Bravo, Cortina, Pacheco, \& Quiñones, 2009).

Bien se sabe que, desde la década de los 50s, cuando se elaboró en Estados Unidos los primeros instrumentos para la medición del ambiente institucional en centros de educación, se vienen obteniendo resultados positivos en las instituciones educativas, por la existencia de planes estratégicos bien definidos que examinan el presente y el futuro de las organizaciones. Veinte años más tarde, la noción se extiende a otros tipos de organizaciones como bancos, escuelas, administración central de gobiernos, agencias de empleo y hospitales que trabajan con instrumentos de evaluación derivados de sus planes estratégicos (Fernández-Romero, 2004).

En efecto, diversos estudios (Ignacio, 2007; Martínez-Vilanova, 2008; Mendoza, 2009; Mastache, 2012; Ramos, 2012; Jimenez, 2015) han encontrado diferentes problemas debido a una mala planificación estratégica que afecta el buen desarrollo de la organización. Esta investigación analiza a una institución privada confesional. Es decir, oficialmente esta institución cuenta con 208,938 miembros afiliados (UPN, 2016). De acuerdo con estas cifras se puede percibir, cuantitativamente, el impacto tan amplio que tiene su plan estratégico. Considerando que ésta es una organización no lucrativa, su interés constante es la efectividad de sus ejes estratégicos evidenciados en la misión, visión, valores, políticas, etcétera; y alcanzar competitividad en su gestión. Esta investigación busca describir ¿cuál es el nivel de cumplimiento del plan estratégico a través del sistema de evaluación REDER de una institución privada confesional, 2017-2019?

\section{Sistema de evaluación REDER}

No se puede hablar del sistema de evaluación REDER, sin antes introducir al Modelo EFQM de excelencia. Este es un enfoque que fue elaborado y diseñado por la European Foundation for Quality Management (de donde proviene sus siglas en inglés, EFQM), la cual fue fundada en 1988 por catorce (14) empresas centroeuropeas líderes en los servicios y la industria. Según el alcance de Moreno (2007) el "modelo EFQM de excelencia se trata de un sistema generalista no vinculado a un sector concreto o actividad". Este modelo utiliza el sistema de gestión basada en procesos. En la actualidad este grupo cuenta con más de 600 miembros, desde pequeñas empresas hasta grandes multinacionales, escuelas de negocios, institutos de investigación y 
universidades. La Fundación Europea para la Gestión de Calidad, en 1992 otorgó ciertos parámetros que fortalecerían en este sentido las organizaciones. Este es un modelo que se fundamenta en ideas de mejora dando el énfasis en los resultados, y la autoevaluación como proceso clave (Campo \& Ortega, 2017). En 1999, el modelo se modificó a uno más avanzado donde se da mayor énfasis a la innovación, al aprendizaje como esencia de una ventaja competitiva (prioridad a los clientes y otros grupos de interés), al desarrollo del conocimiento, entre otros (Khalil, 2013; Machorro, Mercado, Cernas, \& Romero, 2016).

Por consiguiente, el sistema de evaluación REDER nace como una herramienta de evaluación que permite "tener una visión realista de la organización" la cual ayuda a medir de manera cuantitativa a una institución. Este sistema de evaluación es admitido por el mundo organizacional y se apoya en la teoría del Modelo EFQM de excelencia (Moreno, 2007). El sistema de evaluación REDER instituye un conjunto de aspectos a evaluar muy importantes para este modelo. En todo el contexto de su evaluación considera los aspectos más relevantes para el plan estratégico de cualquier organización, sin importar el rubro económico al que éste pertenece (Juaneda, González, \& Marcuello, 2013). Estos son considerados como criterios y subcriterios, los cuales se detallarán:

Resultados u objetivos a alcanzar,

Enfoques o sistemas,

Despliegues,

Evaluación y

Revisión de los enfoques a través de los resultados y las mejoras implantadas.

Esquema de evaluación. Esta es la estructura que ha sido utilizada en el instrumento de este estudio, cuya metodología es la que se recomienda en la "Guía para la evaluación de planes estratégicos en el ámbito universitario”, propuesta por el Grupo de Planificación y Dirección Estratégica de instituciones europeas (GPDE, 2009). A continuación, se detallan sus dimensiones.

a) Liderazgo y bases. El plan estratégico es desarrollado por la alta dirección, en este se desarrolla la misión, visión y valores de la institución, considerando las expectativas de los grupos de interés y haciendo uso de las ventajas competitivas. Constantemente es revisado y evaluado su desarrollo. Para el éxito del plan estratégico, es necesario y fundamental el compromiso de los altos líderes y que a su vez se facilite las herramientas necesarias para su efectivo desarrollo. 
b) Organización. La alta dirección acuerda y aprueba la estructura y el proceso del plan estratégico. Para que estos estándares sean cumplidos a efectividad es necesario la inclusión de grupos de trabajo y equipos responsables, fiables y consistentes, forjando así una comunicación clara a la comunidad empresarial y a los grupos de interés.

c) Análisis y diagnóstico. Para el análisis y diagnóstico, la Institución implanta las bases del plan estratégico, identifica las fortalezas y debilidades de la organización, y las amenazas y las oportunidades del entorno. Por medio de estas es factible obtener la identificación y definición de las ventajas competitivas y los factores críticos de éxito.

d) Formulación. Bajo este enfoque, un plan estratégico considerado excelente formula sus objetivos y estrategias en el análisis y la comprensión de las necesidades y expectativas de los grupos de interés, y las del entorno. Para lograr sus objetivos estratégicos, la Institución formula estrategias que se desarrollarán por medio de planes y programas, para lo cual es necesario la implementación de indicadores, además de un sistema de medición y análisis.

e) Implantación y revisión. Este plan estratégico "se implanta mediante la definición y desarrollo de procesos de comunicación, despliegue, alineación, información, seguimiento y revisión de los citados procesos" (GPDE, 2009, p. 31). La alta dirección conoce los objetivos de la organización y están comprometidos especialmente con los objetivos de los que son responsables. Ellos generan una revisión detallada y diligente del enfoque de tal forma que ésta contribuya a la mejora continua de la organización.

\section{Materiales y métodos}

Se empleó una investigación de tipo descriptiva, porque se buscó describir los niveles, propiedades, características y rasgos importantes de la variable expuesta y sometida al estudio; de corte transversal, porque los datos a analizar se recopilaron en un único momento de tiempo. Adscrito de acuerdo con los protocolos correspondientes a un enfoque cuantitativo. Diseño no experimental (Hernández, Fernández, \& Baptista, 2010). El estudio fue aplicado a 45 líderes de una institución privada confesional, es decir, se consideró el total de la población de estudio en la condición de la muestra.

\section{Instrumento}

El instrumento seleccionado involucra las dimensiones del plan estratégico enfocadas en el sistema de evaluación REDER. El cuestionario comprende una evaluación detallada del plan estratégico, cuya metodología es la que se recomienda en la "Guía para la evaluación de planes estratégicos en el ámbito universitario” propuesta por el Grupo de Planificación y Dirección 
Estratégica de un Foro comprendido por diecisiete (17) Universidades de España (GPDE, 2009).

Tal instrumento no ha sufrido ninguna variación en la redacción de sus reactivos, sólo en la nomenclatura universitaria que se usa en el original, para lo cual se ha reemplazado por una denominación "Institucional", dado que también ha sido elaborado bajo estos estándares. El cuestionario consta de 26 reactivos que pretende medir el nivel de cumplimiento del plan estratégico a través del sistema de evaluación REDER, dividido en 5 secciones: (1) Liderazgo y bases, 6 reactivos; (2) Organización, 5 reactivos; (3) Análisis y diagnóstico, 6 reactivos; (4) Formulación, 4 reactivos; (5) Implantación y revisión, 5 reactivos. El participante asignó a cada reactivo una puntuación del 0 al 100, sólo considerando los múltiplos de 5, cuya interpretación de los resultados ha consistido en los 5 niveles de cumplimiento estandarizados en la metodología de evaluación original: Muy alto, notable, medio, escaso e inexistente.

A su vez, se ha realizado una diligente revisión a la estructura del instrumento utilizado, así como a la teoría de la variable de estudio: plan estratégico; llegando a la conclusión que existe un paralelismo en ambos enfoques, haciendo uso de nomenclaturas diferentes en sus dimensiones. Sin embargo, el instrumento se fundamenta totalmente en la teoría del plan estratégico; por lo tanto, a fin de comprender mejor el paralelismo entre la dimensión teórica e instrumental, se presenta detalladamente en la Tabla 1:

Tabla 1

Paralelismo instrumental y teórico

\begin{tabular}{ll}
\hline Dimensión instrumental & Dimensión teórica de la variable \\
\hline (1) Liderazgo y bases & (1) (2) Análisis estratégico de la situación \\
(2) Organización & (3) Diagnóstico estratégico de la situación \\
(3) Análisis y diagnóstico & (4) Elección de estrategias \\
(4) Formulación & (5) Implantación de estrategias \\
(5) Implantación y revisión & \\
\hline
\end{tabular}

Fuente propia

\section{Resultados}

\section{Niveles de cumplimiento del plan estratégico}

En el contexto del objetivo general, la tabla 2 describe los niveles de cumplimiento del plan estratégico, cuyos niveles son: inexistente, escaso, medio, notable y muy alto. En este sentido, los resultados evidencian que el $40 \%$ del total de la población ubica su percepción en los niveles: "Muy alto" y "Notable", sobre el nivel de cumplimiento del plan estratégico; es decir, la institución ha considerado la misión, visión, valores y el análisis estratégico, así como los 
demás elementos del plan estratégico en su elaboración. Sin embargo, el 35.6\% considera que no se ha dado cumplimiento de estos parámetros.

Tabla 2

Niveles de cumplimiento del plan estratégico

\begin{tabular}{lcc}
\hline & \multicolumn{2}{c}{ Niveles de cumplimiento } \\
\cline { 2 - 3 } & $\mathrm{n}$ & $\%$ \\
\hline Inexistente & 4 & 8.9 \\
Escaso & 12 & 26.7 \\
Medio & 11 & 24.4 \\
Notable & 12 & 26.7 \\
Muy alto & 6 & 13.3 \\
Total & 45 & 100.0 \\
\hline
\end{tabular}

Fuente propia

\section{Niveles de cumplimiento por dimensiones del plan estratégico}

En la Tabla 3 se observa que el 53.4\% se ubica en los niveles: "Escaso" y "Medio", cuyos niveles describen el cumplimiento de su liderazgo y bases; es decir, se evidencia poca existencia en el análisis estratégico de la situación; asimismo, se describe que los grupos de interés participan en grupos de trabajo sobre el diseño y definición del plan estratégico, dejando la evidencia de un enfoque proactivo en la definición de misión, visión, valores y objetivos estratégicos.

Por otro lado, el $13.3 \%$ de los participantes describen que esta dimensión evaluada se encuentra en el nivel: "Muy alto"; es decir, que posee un despliegue completo y sistemático del enfoque, además da evidencia que se evalúa el impacto de los riesgos, los resultados de la información y prospectiva, bajo los mecanismos de identificación de los factores críticos de éxito y sus ventajas competitivas.

Tabla 3

Niveles de cumplimiento de liderazgo y bases

\begin{tabular}{lcc} 
& \multicolumn{2}{c}{ Liderazgo y bases } \\
\cline { 2 - 3 } & $\mathrm{n}$ & $\%$ \\
\hline Inexistente & 4 & 8.9 \\
Escaso & 12 & 26.7 \\
Medio & 12 & 26.7 \\
Notable & 11 & 24.4 \\
Muy alto & 6 & 13.3 \\
Total & 45 & 100.0 \\
\hline
\end{tabular}

Fuente propia 
Se observa y se describe, en la tabla 4, el nivel muy alto de cumplimiento, el cual es respaldado por el $13.3 \%$ de los participantes; significa que, bajo su enfoque organizativo, el análisis estratégico de la situación sufre una revisión cada fin de periodo, analizando la eficacia del enfoque y su despliegue obteniendo mejoras continuas, las cuales no son sino consecuencia de aquellas revisiones. Por otro lado, se describe que el 35.6\% de los participantes, se ubica en los niveles: "Escaso" e "Inexistente"; describe que se han definido parcialmente los procedimientos y, en algunos casos, existen procesos que no han sido aprobados por la administración, además existen apenas evidencias escasas de los mecanismos sistematizados de comunicación interna y externa en la institución.

Tabla 4

Niveles cumplimiento de organización

\begin{tabular}{lcc}
\hline & \multicolumn{2}{c}{ Organización } \\
\cline { 2 - 3 } & $\mathrm{n}$ & $\%$ \\
\hline Inexistente & 4 & 8.9 \\
Escaso & 12 & 26.7 \\
Medio & 11 & 24.4 \\
Notable & 12 & 26.7 \\
Muy alto & 6 & 13.3 \\
Total & 45 & 100.0 \\
\hline
\end{tabular}

Fuente propia

En la tabla 5, se describe que el porcentaje más alto se centra en un nivel "Notable" de cumplimiento, cuyo porcentaje es el $28.9 \%$, indicando que para la dimensión diagnóstico estratégico de la situación existe un procedimiento adecuado, identificándose las fortalezas, oportunidades, debilidades y amenazas de la institución, revisando la eficacia del proceso, además de la existencia de un proceso formal documentado con criterios definidos sobre el objetivo y alcance de la vigilancia competitiva, evidenciando así un despliegue completo y alcance total de los factores críticos de éxito. A su vez, el 35.6\% de los participantes aseguraron que esta dimensión reposa entre los niveles: "Escaso" e "Inexistente" de cumplimiento; referenciando pocas evidencias de actividades sobre la vigilancia competitiva y, que, además, no se han analizado completamente los aspectos políticos, tecnológicos, económicos y sociales para la elaboración de la matriz FODA.

Tabla 5

Niveles de cumplimiento de análisis y diagnóstico

\begin{tabular}{ccc}
\hline & \multicolumn{2}{c}{ Análisis y diagnóstico } \\
\cline { 2 - 3 } & $\mathrm{n}$ & $\%$ \\
\hline Inexistente & 4 & 8.9 \\
\hline
\end{tabular}




\begin{tabular}{lcc}
\hline Escaso & 12 & 26.7 \\
Medio & 11 & 24.4 \\
Notable & 13 & 28.9 \\
Muy alto & 5 & 11.1 \\
Total & 45 & 100.0 \\
\hline
\end{tabular}

Fuente propia

En esta sección se evidencia que el $40 \%$ de los participantes describe a la institución con niveles de cumplimiento entre "Notable" y "Muy alto", revelando el estado de la elección y formulación de estrategias, considerando que los objetivos estratégicos han facilitado su evaluación posterior de forma ágil y sistemática. Además, se ha usado herramientas para llevar acabo la misión, avanzando de forma sistémica hacia la visión organizacional; también se demostró el uso de calendarios de implantación, desarrollo, ejecución de planes y programas. Por otro lado, el $8.9 \%$ de la población percibe que no existen evidencias de cumplimiento en la evaluación de esta dimensión, tal como lo revela la tabla 6 .

Tabla 6

Niveles de cumplimiento de formulación

\begin{tabular}{lcc}
\hline & \multicolumn{2}{c}{ Formulación } \\
\cline { 2 - 3 } & $\mathrm{n}$ & $\%$ \\
\hline Inexistente & 4 & 8.9 \\
Escaso & 13 & 28.9 \\
Medio & 10 & 22.2 \\
Notable & 13 & 28.9 \\
Muy alto & 5 & 11.1 \\
Total & 45 & 100.0 \\
\hline
\end{tabular}

Fuente propia

En la tabla 7, se describe el nivel de cumplimiento en la implantación y revisión de estrategias; demostrando que el $11.1 \%$ de la población de estudio percibe que existe un nivel "Muy alto" de cumplimiento; lo que quiere decir que se revisa al final de cada periodo la eficacia del plan estratégico y se introducen mejoras en el mismo, por efecto y consecuencias de estas revisiones; también se integran los planes específicos en el plan institucional y se mejora periódicamente la política y estrategia. En el extremo, se encuentran quienes aseguran la inexistencia de un nivel de cumplimiento en la organización, representados por el $8.9 \%$ de la población; es decir, para estos participantes no se difunde ni se despliega el plan estratégico lo suficiente.

Tabla 7

Niveles de cumplimiento de implantación y revisión 


\begin{tabular}{lcc}
\hline & \multicolumn{2}{c}{ Implantación y revisión } \\
\cline { 2 - 3 } & $\mathrm{n}$ & $\%$ \\
\hline Inexistente & 4 & 8.9 \\
Escaso & 12 & 26.7 \\
Medio & 12 & 26.7 \\
Notable & 12 & 26.7 \\
Muy alto & 5 & 11.1 \\
Total & 45 & 100.0 \\
\hline
\end{tabular}

Fuente propia

\section{Discusión}

De acuerdo con los resultados encontrados en este estudio se alude que las investigaciones analizadas han hecho uso del modelo EFQM de excelencia, con un enfoque de evaluación y validación en la gestión empresarial haciendo énfasis en ciertos ejes estratégicos; sin embargo, no se evidencia que hayan empleado el sistema de evaluación REDER a sus planes estratégicos.

En sus respectivas investigaciones, Ignacio (2007), Martínez-Vilanova (2008) y Mastache (2012) hacen referencia a la validez de este enfoque en sus resultados. Cabe mencionar que Ignacio (2007) al hacer uso del modelo EFQM de excelencia impulsó la calidad en sus procesos y el desarrollo de los objetivos estratégicos. Sin embargo, después de haber hecho una escudriñada revisión a los estudios de Mendoza (2009), se puede concluir que su enfoque integrador de estrategias también ha causado un efecto positivo en la gestión organizacional; además de la mejora en otros indicadores estratégicos a la institución. Si bien es cierto, estos autores hicieron uso del enfoque que da soporte a este estudio, no hay evidencia de la aplicación de algún sistema de evaluación antes, durante o después de la ejecución de su plan estratégico.

Por otro lado, luego de haber realizado una revisión diligente a estudios previos ya mencionados anteriormente, se halló que uno de los resultados de la presente investigación guarda congruencia al estudio de Ramos (2012), Jiménez (2015) y Campos (2014), lo cuales enfocaron un sistema de control sobre la planificación estratégica, en sus estudios evidencian que el sistema de seguimiento y evaluación que utilizaron para verificar la efectividad y cumplimiento del plan estratégico, brindó los resultados organizacionales que esperaron. Sin embargo, es necesario aclarar que ninguno de estos investigadores ya mencionados ha tenido el objetivo de medir el nivel de cumplimiento del plan estratégico a través del sistema de evaluación REDER, el cual es recomendado por el modelo EFQM de excelencia.

A su vez, los resultados de la presente investigación están en total congruencia con los aportes científicos otorgados por Jiménez (2015), donde uno de sus resultados con mayor 
relevancia fue el establecimiento de una metodología que garantizó la emisión de alertas tempranas para la formulación de acciones preventivas y correctivas dentro del ciclo de ejecución de la estrategia, con la finalidad de instrumentar el proceso de seguimiento y evaluación del cumplimiento en la planificación institucional. Otro de los resultados más notables fue documentar que la máxima autoridad de la organización debe establecer los lineamientos claros para la ejecución del plan estratégico en todos los niveles jerárquicos y en todas sus etapas desde la preparación hasta su cierre.

En consecuencia, se evidenció que el nivel de cumplimiento del plan estratégico a través del sistema de evaluación REDER de una institución privada confesional, 2017-2019, se ubica por debajo de los estándares medios. A pesar de no encontrarse estudios previos en los cuales se haya usado el sistema de evaluación REDER a un plan estratégico convencional, su aplicación fue efectiva. Siendo que su metodología es adaptable a cualquier organización, aun cuando ésta sea considerada sin fines de lucro.

\section{Referencias}

Aguirre, J. (2015). "Inteligencia estratégica: un sistema para gestionar la innovación". Estudios Gerenciales, 31, 100-110. doi:https://doi.org/10.1016/j.estger.2014.07.001

Araya, A. (2017). "Modelos de planeación estratégica en las empresas familiares". TEC Empresarial, XI(1), 23-34. Obtenido de http://www.scielo.sa.cr/pdf/tec/v11n1/16593359-tec-11-01-00023.pdf

Caballero, S., Canchucaja, P., Miguel, J., \& Paitamala, O. (2012). Plan Estratégico del Sector Artesanal de la Región Junín. Huancayo: Pontificio Universidad Católica del Perú.

Campo, S., \& Ortega, C. (2017). Validación del cuestionario de autoevaluación para unidades administrativas y de servicios. Ingeniare. Revista chilena de ingeniería, 25(2), 338349. Obtenido de https://scielo.conicyt.cl/pdf/ingeniare/v25n2/0718-3305-ingeniare25-02-00338.pdf

Campos, F. (2014). Aplicación de un sistema de planificación estratégica y control de gestión en una organización sin fines de lucro: Caso ONG Psicólogos voluntarios. Santiago: Universidad de Chile. Recuperado el 24 de Septiembre de 2017, de http://repositorio.uchile.cl/bitstream/handle/2250/117539/Tesis\%2023082014\%20Fab i\%C3\%A1n\%20Campos.pdf?sequence=1

Carponi, J. C. (2012). Recuperado el 10 de Octube de 2012, de http://www.clublanus.com/files/pdf/planificacionestrategica.pdf

Cova, L., \& Moreno, L. (2010). Diseño de un Plan Estratégico para el mejoramiento de las actividades de una Microempresa ubicada en la Vía Alterna Barcelona - Puerto la Cruz, Estado Anzoátegui. Barcelona: Universidad del Oriente nucleo de Anzoátegui. Recuperado el 08 de Marzo de 2016, de http://ri.bib.udo.edu.ve/bitstream/123456789/2896/1/15-TESIS.IS010C27.pdf 
Cruz, R. (2010). Técnicas Cuantitativas para la evaluación en la Planeación. Veracruz: Universidad Veracruzana - Facultad de Contaduría y Administración.

Echeverría, R. (2014). Modelo de Planificación Operativa por procesos para la Gestión Administrativa interna de la Asamblea Nacional del Ecuador. Quito-Ecuador: Universidad Tecnológica Israel.

Escudero, F. (2003). Planificación operacional y financiera en la hotelería. México D. F.: Editorial Trillas S.A. de C.V.

Fernández, J., Barbón, O., \& Añorga, J. (2016). Enfoque estratégico en la gestión de las universidades y la concepción estratégica de la Educación Avanzada. Revista Cubana de Reumatología, 18(1), 71-75. Obtenido de http://scielo.sld.cu/pdf/rcur/v18n1/rcur12116.pdf

Fernández-Romero, A. (2004). Dirección y planificación estratégicas en las empresas y organizaciones. Madrid: Ediciones Dias de Santos SA.

Flores, I., \& Flores, R. (2016). El proceso de planificación y el presupuesto gubernamental. México: Universidad Autónoma del Estado de Hidalgo. Recuperado el 2016, de http://www.uaeh.edu.mx/investigacion/estl/LI_AdmninEst/Ruth_Flores/articulo_plani ficacion.pdf

Gálvez, D. (2010). Modelo de Planeación Estratégica aplicado a una microempresa artesanal del sector metalmecánico del Ecuador, caso empresa metalmecánica Gálvez EMG Hierro Forjado. Quito: Escuela Politécnica Nacional.

Gonzales, L., \& Gonzales, M. (2017). Gestión estratégica de la Comunidad Colombiana de Cómputo Avanzado $3 \mathrm{CoA} \circledast$ mediante análisis DOFA y cocreación. Ingeniare. Revista chilena de ingeniería, 25(3), 464-476. doi:http://dx.doi.org/10.4067/S071833052017000300464

GPDE. (2009). Guía para la evaluación de Planes Estratégicos en el ámbito Universitario. Grupo de Planificación y Dirección Estratégica. España: Foro de Universidades. Recuperado el 2 de Noviembre de 2016, de http://acdc.sav.us.es/jpe/images/PPTs/gua\%20para\%20la\%20evaluacin\%20de\%20pla nes $\% 20$

Gutiérrez, J., Alizo, M., Morales, M., \& Romero, J. (2016). Planificación estratégica situacional: Perspectiva de una unidad científica universitaria. Revista Venezolana de Gerencia, XXI(76), 607-626. Obtenido de http://www.redalyc.org/pdf/290/29049487003.pdf

Hernández, R., Fernández, C., \& Baptista, P. (2010). Metodología de la Investigación (Cuarta ed.). México DF: McGRAW HILL INTERAMERIC MA EDITORES SA DE C.V.

Ignacio, E. (2007). Validación de un modelo para medir la calidad asistencial en los hospitales. Cádiz: Universidad de Cádiz. 
Jaimes, H., Bravo, S., Cortina, A., Pacheco, C., \& Quiñones, M. (2009). Planeación estratégica de largo plazo: una necesidad de corto plazo. Pensamiento \& Gestión(25), 191-213. Obtenido de http://www.scielo.org.co/pdf/pege/n26/n26a09.pdf

Jiménez, J. (2015). Desarrollo de la planificación estratégica y diseño de un sistema de control y evaluación de gestión institucional de EMASEO EP. para el período 2015 2018. Quito-Ecuador: Universidad Internacional del Ecuador. Recuperado el 24 de Septiembre de 2017, de http://repositorio.uide.edu.ec/bitstream/37000/905/1/T-UIDE1031.pdf

Juaneda, E., González, L., \& Marcuello, C. (2013). El reto de la calidad para el Tercer Sector Social. Análisis de casos de implantación del modelo EFQM. Cuadernos de Gestión, 13(2), 111-126. doi:10.5295/cdg.110285ea

Khalil, A. (2013). Construcción de un modelo de evaluación de la calidad de la enseñanza universitaria desde el punto de vista de los alumnos. Córdova: Universidad de Córdova. Facultad de Ciencias de la Educación.

León, C. (2011). Diseño de una Planificación Estratégica para la empresa Reprodata Cia. Ltda. dedicada a la reparación y mantenimiento de equipos de copiado e impresión (impresoras y multifunciones) marca Lexmark, de la ciudad de Quito. Quito: Universidad Politécnica Salesiana.

López, E. (2004). Objetivos Organizacionales. Obtenido de Objetivos Organizacionales: http://www.monografias.com/trabajos 15/objetivos-organizacionales/objetivosorganizacionales.shtml

Machorro, F., Mercado, P., Cernas, D., \& Romero, M. (2016). Influencia del capital relacional en el desempeño organizacional de las instituciones de educación superior tecnológica. Revista Innovar Journal, 26(60), 35-50. doi:http://dx.doi.org/10.15446/innovar.v26n60.55531

Martelo, R., Ponce, A., \& Acuña, F. (2016). Guía Metodológica para el Diseño de un Plan Estratégico Informático en Instituciones de Educación Superior. Formación Universitaria, IX(1), 91-98. doi:10.4067/S0718-50062016000100010

Martínez, D., \& Milla, A. (2005). La Elaboración del Plan Estratégico y su Implantación a través del Cuadro de Mando Integral. España: Ediciones Días de Santos.

Martinez-Vilanova, A. M. (2008). Modelo de evaluación y diagnosis de excelencia en la gestión, basado en el Cuadro de Mando Integral y el Modelo EFQM de Excelencia®. Aplicación a las Cajas Rurales. Valencia: Universidad Politécnica de Valencia.

Mastache, A. (2012). Implantación del modelo EFQM y su impacto en la competitividad de la empresa: Un análisis de casos de empresas localizadas en Asturias. Ovideo: Universidad de Ovideo.

Mendoza. (2009). Uso del Modelo EFQM de Excelencia como instrumento de evaluación del desempeño empresarial de la Clínica Ejido C.A. (Una propuesta de mejora). Mérida: Universidad de los Andes - Venezuela. 
Mendoza, D., \& López, D. (2015). Pensamiento Estratégico: Centro neurálgico de la planificación estratégica que transforma la visión en acción . Económicas CUC, 36(1), 81-94. Obtenido de https://dialnet.unirioja.es/descarga/articulo/5156212.pdf

Mendoza, D., López, D., \& Salas, E. (2016). Planificación estratégica de recursos humanos: efectiva forma de identificar necesidades de personal. Económicas CUC, 31(1), 6178. doi:http://dx.doi.org/10.17981/econcuc.15.5.2016.03

Montoya, I. (2009). La Formación de la Estrategia en Mintzberg y las posibilidades de su aportación para el futuro. Universidad Nacional de Colombia, 31.

Mora-Riapira, E., Vera-Colina, M., \& Melgarejo-Molina, Z. (2015). Planificación estratégica y niveles de competitividad de las Mipymes del sector comercio en Bogotá. Estudios Gerenciales, 31, 79-87. doi:https://doi.org/10.1016/j.estger.2014.08.001

Moreno, J. (2007). Guía para la aplicación del Modelo EFQM de excelencia en entidades de acción social. España: Fundación Luis Vives.

Nava, R. (2009). La Planificación Operativa y las técnicas de presupuesto por programa y por proyecto. Análisis Comparativo. Mérida: Universidad de los Andes.

Paredes, N. (2003). Enfoque del Planeamiento Estratégico para la empresa gráfica de la UNMSM. Lima: Universidad Nacional Mayor de San Marcos.

Pérez-Vallejo, L., Vilariño-Corella, C., \& Ronda-Pupo, G. (2016). El cambio organizacional como herramienta para coadyuvar la implementación de la estrategia. Ingeniería Industrial, 37(3), 286-294. Obtenido de http://scielo.sld.cu/pdf/rii/v37n3/rii070316.pdf

Ramos, H. (2012). Propuesta de un modelo de gestión para mejorar la dirección municipal de La Provincia de Otuzco. Producción y Gestión, 16(1), 35-44. Recuperado el 10 de Enero de 2017

Romo, G., \& Márquez, E. (2014). Gestión estratégica vs. Análisis estratégico. Una discusión conceptual a partir del estudio de caso de una institución de educación superior. Pensamiento y Gestión(36), 267-290. Obtenido de http://www.scielo.org.co/pdf/pege/n36/n36a10.pdf

Sallenave, J.-P. (2002). Gerencia Integral. Bogotá: Editorial Norma S.A.

Sánchez, I. (Mayo de 2017). "La planeación estratégica en el Sistema de Salud cubano". MEDISAN, 21(5), 635-641. Obtenido de http://scielo.sld.cu/pdf/san/v21n5/san19215.pdf

Sierra, E. (1978). Planificación operativa y el plan de mediano plazo. Comercio Exterior, 28(4), 394-400.

Union Peruana del Norte. (2016). Informe de actualidad de Feligresía. Lima: Iglesia Adventista del Séptimo Día. 
Valencia, G., \& Alfonso, M. (2016). El reto de la planificación estratégica en las Pymes. Revista Publicando, III(8), 335-344. Obtenido de https://dialnet.unirioja.es/descarga/articulo/5833399.pdf

Villalva, J. (2009). Etapas del proceso administrativo. Argentina: El Cid Editor | apuntes.

Zavala-Mendoza, E., \& Varela, A. (2017). Planificación estratégica en la organización y gestión de servicios sociales: la transformación administrativa en el trabajo social. Voces desde el trabajo social, V(1), 69-98. doi:https://doi.org/10.31919/voces.v5i1.80 